\title{
Measurement of fission yields and isomeric yield ratios at IGISOL
}

\author{
Stephan Pomp ${ }^{1, \star}$, Andrea Mattera ${ }^{1}$, Vasileios Rakopoulos ${ }^{1}$, Ali Al-Adili ${ }^{1}$, Mattias Lantz ${ }^{1}$, Andreas \\ Solders ${ }^{1}$, Kaj Jansson ${ }^{1}$, Alexander V. Prokofiev ${ }^{1}$, Tommi Eronen ${ }^{2}$, Dimitri Gorelov², Ari Jokinen², \\ Anu Kankainen², lain D. Moore ${ }^{2}$, Heikki Penttilä ${ }^{2}$, and Sami Rinta-Antila ${ }^{2}$ \\ ${ }^{1}$ Uppsala University, Department of Physics and Astronomy, Box 516, 75120 Uppsala, Sweden \\ ${ }^{2}$ University of Jyväskylä, Department of Physics, P.O. Box 35 (YFL), 40014 Jyväskylä, Finland
}

\begin{abstract}
Data on fission yields and isomeric yield ratios (IYR) are tools to study the fission process, in particular the generation of angular momentum. We use the IGISOL facility with the Penning trap JYFLTRAP in Jyväskylä, Finland, for such measurements on ${ }^{232} \mathrm{Th}$ and ${ }^{\text {nat }} \mathrm{U}$ targets. Previously published fission yield data from IGISOL concern the ${ }^{232} \mathrm{Th}(\mathrm{p}, \mathrm{f})$ and ${ }^{238} \mathrm{U}(\mathrm{p}, \mathrm{f})$ reactions at 25 and $50 \mathrm{MeV}$. Recently, a neutron source, using the $\mathrm{Be}(\mathrm{p}, \mathrm{n})$ reaction, has been developed, installed and tested. We summarize the results for $(\mathrm{p}, \mathrm{f})$ focusing on the first measurement of IYR by direct ion counting. We also present first results for IYR and relative yields for $\mathrm{Sn}$ and $\mathrm{Sb}$ isotopes in the 128-133 mass range from ${ }^{n a t} \mathrm{U}(\mathrm{n}, \mathrm{f})$ based on $\gamma$-spectrometry. We find a staggering behaviour in the cumulative yields for $\mathrm{Sn}$ and a shift in the independent fission yields for $\mathrm{Sb}$ as compared to current evaluations. Plans for the future experimental program on fission yields and IYR measurements are discussed.
\end{abstract}

\section{Introduction}

Fission yields (FY) are a key observable for studies of the fission process. They are measured for example using the $2 \mathrm{E}$ or the $2 \mathrm{E}-2 \mathrm{v}$-technique [1], techniques involving conventional mass spectrometers like LOHENGRIN [2,3] or using irradiation techniques in combination with $\gamma$-spectrometry [4]. The challenges in the mentioned techniques are connected to the achievable mass resolution (2E and $2 \mathrm{E}-2 \mathrm{v}$ technique), limits in the achievable excitation energies in the fissioning nucleus (in the case of LOHENGRIN), or the necessity of sufficiently long life times of the studied fission products in combination with the requirement of well-known decay data (when using $\gamma$-spectrometry).

An alternative method to measure fission yields is provided by the usage of the ISOL-technique (Isotope Separation On-Line) in combination with a Penning trap for mass separation. With this approach independent isotopic FY can be measured for a range of fissioning systems with different excitation energies and, since the method is based on direct ion counting, without requirements on $\gamma$-decay schemes.

Furthermore, the high mass resolving power provided by Penning traps make it possible to measure isomeric yield ratios (IYR). This is then, again, not based on the measurement of gamma decays

^e-mail: stephan.pomp@physics.uu.se 
but on direct ion counting, resolving the ground state mass from the mass of the isomeric state. Routinely achievable mass resolving powers correspond to a resolution of about $0.5 \mathrm{MeV}$ in excitation energy. New developments like the Phase-Imaging Ion-Cyclotron-Resonance technique [5] can achieve a resolution of $50 \mathrm{keV}$. This opens the door to both high-quality FY data and measurements of a wide range of IYR for various fission systems.

The IYR may be used to deduce the angular momentum distributions of the fission fragments. This allows thus to study the amount of energy available in the fission process that is converted into rotational energy. The initial spin also influences the production of fission neutrons and gamma rays in the de-excitation process of the fragment [6]. Therefore, IYR, measured for a range of fissioning systems and across all masses, provide important information and constraints for modelling the fission process and its dynamics.

It has also been pointed out that the differences between calculated and measured antineutrino production at Daya Bay may be due to a lack of knowledge on IYR [7,8].

Last but not least, it should be mentioned that, due to high N/Z ratio and low average promptneutron emission multiplicity, neutron-induced fission of ${ }^{232} \mathrm{Th}$ and ${ }^{238} \mathrm{U}$ is an efficient way to produce exotic nuclei. Using a neutron-production target within an ISOL facility and the ${ }^{232} \mathrm{Th}(\mathrm{n}, \mathrm{f})$ reaction is the most promising way to produce very neutron-rich nuclei especially in the light mass region, close to ${ }^{78} \mathrm{Ni}$, for further studies with a Penning trap.

\section{Experimental}

The measurements discussed here have been performed at the IGISOL facility, located at the Accelerator Laboratory of the University of Jyväskylä, Finland. Here we give a brief outline of the facility. Details can be found in Refs. $[9,10]$.

The K-130 cyclotron delivers a proton beam to the fission chamber. In the presented cases this beam is used for both $(p, f)$ and $(n, f)$ reaction studies. In the first case a $25 \mathrm{MeV}$ beam directly interacts with ${ }^{232} \mathrm{Th}$ or ${ }^{\text {nat }} \mathrm{U}$ targets to induce (p,f) reactions. In the (n,f) case, a $30 \mathrm{MeV}$ beam is used to create neutrons in a thick Beryllium target via the $\mathrm{Be}(\mathrm{p}, \mathrm{n})$ reaction. The Be target is located inside the fission chamber and the neutrons then induce $(\mathrm{n}, \mathrm{f})$ reactions in the ${ }^{\text {nat }} \mathrm{U}$ target located a few $\mathrm{cm}$ downstream the neutron production target. Further details on the neutron converter are given below and in Ref. [11].

Helium is used as buffer gas to slow down the fission products and flush them towards a radiofrequency (RF) ion guide where they are stepwise accelerated to $30 \mathrm{kV}$ towards a magnetic mass separator. The time spent by the ions in the buffer gas until extraction towards the magnet is on the order of a few tens of milliseconds.

The ions produced in this way are dominantly in the $1+$ charge state. The relative fraction of ions in the 1+ state may vary from element to element in a non-trivial way and can be measured in dedicated studies. An example is given below. Details of the behaviour of the target cell that is used in the $(\mathrm{p}, \mathrm{f})$ case, i.e., the stopping efficiency for the different ions and potential bias for different fission product masses or their total kinetic energies (TKE), have been studied in Ref. [12]. It was found that such dependences are weak and the associated uncertainties are small compared to other systematic uncertainties. A similar study for the $(\mathrm{n}, \mathrm{f})$ case is currently in progress.

After extraction from the fission chamber by the ion guide and a second vacuum chamber with extraction electrodes, a dipole magnet is used to select ions for an isobaric mass chain. The mass resolving power, $M / \Delta M$ of the magnet is about 500 .

After the magnet, the ions pass through a switch yard where they can either be deflected to a $\gamma$-ray measurement station, or towards the radiofrequency cooler and buncher (RFQ). After deceleration and cooling, short ion bunches of about $10 \mu$ s duration are finally released towards a setup of two 


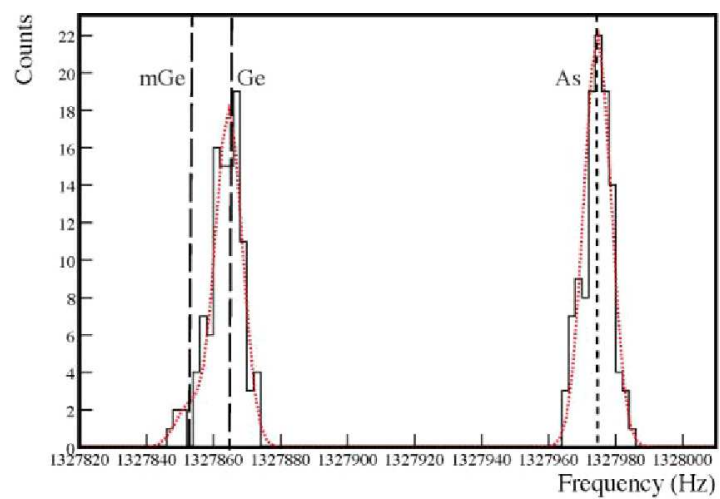

Figure 1. Experimental frequency distribution spectrum for the region of mass $A=81$. The identification of the peaks is based on a frequency to mass calibration. The figure shows the positions of ${ }^{81} \mathrm{As},{ }^{81} \mathrm{Ge}$, and ${ }^{81 m} \mathrm{Ge}$ (dashed black), and the Gaussian fits used to extract the IYR (dotted red).

consecutive Penning traps, JYFLTRAP. In the traps the ions are stored inside a magnetic field and their eigenmotion is manipulated by applying a RF field that selects different $q / m$ ratios in the static $7.0 \mathrm{~T}$ magnetic field. The selected ions then pass through a $1.5 \mathrm{~mm}$ wide aperture and are extracted towards a multichannel plate (MCP). The last step allows for additional time-of-flight measurements which can supress impurities with the same $q / m$ ratio. The total time from production of the fission product until registration on the MCP varies depending on the setting of the experiment, e.g., collection and cooling times in the RFQ. These settings can be changed depending on the measurement needs, e.g., desired mass resolving power. Typically this total time is on the order of a few hundred ms. Therefore, decay corrections have to be applied to the raw data.

The case of ${ }^{81} \mathrm{Ge}$ is the most difficult measurement discussed in this paper. The mass difference between isomer and ground state is only $679 \mathrm{keV}$ and the IYR is small. The isomeric state thus shows up as a small bump almost completely immersed in the mass peak related to the ground state of ${ }^{81} \mathrm{Ge}$. Peak positions are calculated relative to a reference peak, ${ }^{81} \mathrm{As}$ in this case, and all peaks are fitted with Gaussian distributions (see Fig. 1).

It turns out that applying Gaussian fits does not always reproduce the experimental data sufficiently well and the peaks might have systematic distortions depending on settings of the Penning trap. While this has, in general, a negligible effect on the IYR, it is desirable to apply the correct shapes in the fitting process, especially in cases with overlapping peaks. To this end, a method using Gaussian processes to fit imperfect models with non-linear least squares and using a prior distribution has been developed in Uppsala [13]. The method has, however, not yet been applied in the results presented in this paper.

\section{Results and discussion}

\subsection{Proton-induced fission at $25 \mathrm{MeV}$}

Over the past years a series of measurements of FY from the (p,f) reaction on ${ }^{232} \mathrm{Th}$ and ${ }^{n a t} \mathrm{U}$ have been performed by the IGISOL group [14].

From some of these data IYR could be extracted for six nuclei, each from the two fissioning systems. These measurements represent the first IYR obtained by direct ion counting. Furthermore, nine 
Table 1. IYR for the six studied nuclei from (p,f) at $25 \mathrm{MeV}$ for the two studied fissioning systems. Uncertainties are statistical only. The IYR are given as $\mathrm{Ym} /(\mathrm{Ym}+\mathrm{Yg} . \mathrm{s}$.$) .$

\begin{tabular}{|l|l|l|l|c|c|c|}
\hline & $\begin{array}{l}\text { Spin } \\
\text { g.s. }\end{array}$ & $\begin{array}{l}\text { Spin } \\
\text { isom. }\end{array}$ & $\begin{array}{l}\Delta M \\
(\mathrm{keV})\end{array}$ & $\begin{array}{l}\text { IYR for }{ }^{n a t} \mathrm{U} \\
\text { from }[17]\end{array}$ & IYR for ${ }^{\text {nat }} \mathrm{U}$ & IYR for ${ }^{232} \mathrm{Th}$ \\
\hline${ }^{81} \mathbf{G e}$ & $\left(9 / 2^{+}\right)$ & $\left(1 / 2^{+}\right)$ & 679 & - & $\mathbf{0 . 0 3} \pm \mathbf{0 . 0 1}$ & $\mathbf{0 . 0 8} \pm \mathbf{0 . 0 2}$ \\
\hline${ }^{96} \mathbf{Y}$ & $0^{-}$ & $8^{+}$ & 1140 & - & $\mathbf{0 . 5 5} \pm \mathbf{0 . 0 1}$ & $\mathbf{0 . 6 1} \pm \mathbf{0 . 0 2}$ \\
\hline${ }^{97} \mathbf{Y}$ & $\left(1 / 2^{-}\right)$ & $(9 / 2)^{+}$ & 667 & 0.71 & $\mathbf{0 . 7 3} \pm \mathbf{0 . 0 2}$ & $\mathbf{0 . 7 8} \pm \mathbf{0 . 0 2}$ \\
\hline${ }^{128} \mathbf{S n}$ & $0^{+}$ & $\left(7^{-}\right)$ & 2091 & 0.52 & $\mathbf{0 . 5 8} \pm \mathbf{0 . 0 2}$ & $\mathbf{0 . 4 6} \pm \mathbf{0 . 0 1}$ \\
\hline${ }^{129} \mathbf{S b}$ & $7 / 2^{+}$ & $\left(19 / 2^{-}\right)$ & 1851 & - & $\mathbf{0 . 4 7} \pm \mathbf{0 . 0 4}$ & $\mathbf{0 . 4 6} \pm \mathbf{0 . 0 1}$ \\
\hline${ }^{130} \mathbf{S n}$ & $0^{+}$ & $\left(7^{-}\right)$ & 1946 & 0.34 & $\mathbf{0 . 5 4} \pm \mathbf{0 . 0 2}$ & $\mathbf{0 . 3 8} \pm \mathbf{0 . 0 1}$ \\
\hline
\end{tabular}

out of the twelve measurements are the first IYR measurements for the studied system. Preliminary results were presented recently [15]. Here we briefly summarize revised results. A more detailed publication is in progress [16].

In Table 1 we give the spin assignments for the six nuclei in their ground state and their studied isomer, their mass difference, and our measured IYR with their statistical uncertainties. Data from the only other measurement of IYR in the (p,f) reaction obtained by Tanikawa et al. [17] by means of $\gamma$-spectrometry are also included in the table. The IYR are given as the yield for the metastable state over the total yield.

The ${ }^{81} \mathrm{Ge}$ case is the only one out of the six cases where the isomeric state has lower spin compared to the ground state. This is consistent with the comparatively low observed IYR. When comparing the two Yttrium cases, we note that ${ }^{96} \mathrm{Y}$ shows a larger difference in spin between ground state and isomer than ${ }^{97} \mathrm{Y}$ and has as small IYR. In the case of ${ }^{128} \mathrm{Sn}$ and ${ }^{130} \mathrm{Sn}$ we find that, at least for the ${ }^{n a t} \mathrm{U}$ reaction, similar IYR may be expected since ground state and isomeric state have the same spin assignments in both cases. These observations indicate that our results are consistent with each other. Our data are in reasonable agreement with the data from Tanikawa et al. [17] except in the ${ }^{130} \mathrm{Sn}$ case where Ref. [17] gives a value that is much lower and closer to our result for the Thorium case but also much lower than their IYR for ${ }^{128} \mathrm{Sn}$.

Our most striking observation is related to differences in the IYR between the two studied fissioning systems. We see a tendency that the ${ }^{232} \mathrm{Th}(\mathrm{p}, \mathrm{f})$ reaction gives higher IYR, compared to the ${ }^{n a t} \mathrm{U}(\mathrm{p}, \mathrm{f})$ reaction, for nuclei in the light-mass region whereas the opposite trend is seen in the heavymass region. Most notably the IYR for ${ }^{128} \mathrm{Sn}$ and ${ }^{130} \mathrm{Sn}$ are significantly lower for ${ }^{232} \mathrm{Th}(\mathrm{p}, \mathrm{f})$ compared to ${ }^{\text {nat }} \mathrm{U}(\mathrm{p}, \mathrm{f})$. A possible explanation for this observation might be linked to different scission configurations due to differences in fission mode ratios; for ${ }^{232} \mathrm{Th}$ the super-long mode is found to be stronger compared to ${ }^{238} \mathrm{U}$ at comparable excitation energies in the compound system [18].

\subsection{Relative yields and isomeric ratios for $(n, f)$ reactions}

The neutron-induced fission data have been obtained using a thick $(6 \mathrm{~mm})$ Beryllium target. This implies only minor differences in the energy spectra compared to the characterized 5-mm thick watercooled Be target $[11,19]$. The idea behind the development of this target is motivated by the newly installed MCC30/15 cyclotron that is designed to deliver a $30 \mathrm{MeV}$ proton beam with currents up to $100 \mu \mathrm{A}$. This would lead to a power of $3 \mathrm{~kW}$ on the target that needs to be cooled, but could also be used to create a neutron flux that induces a fission rate that is high enough to ensure reasonable measurement times for IFY measurements over a wide mass range. Characterization campaigns at The Svedberg Laboratory in Uppsala and in Jyväskylä showed that the design goal of reaching a total 

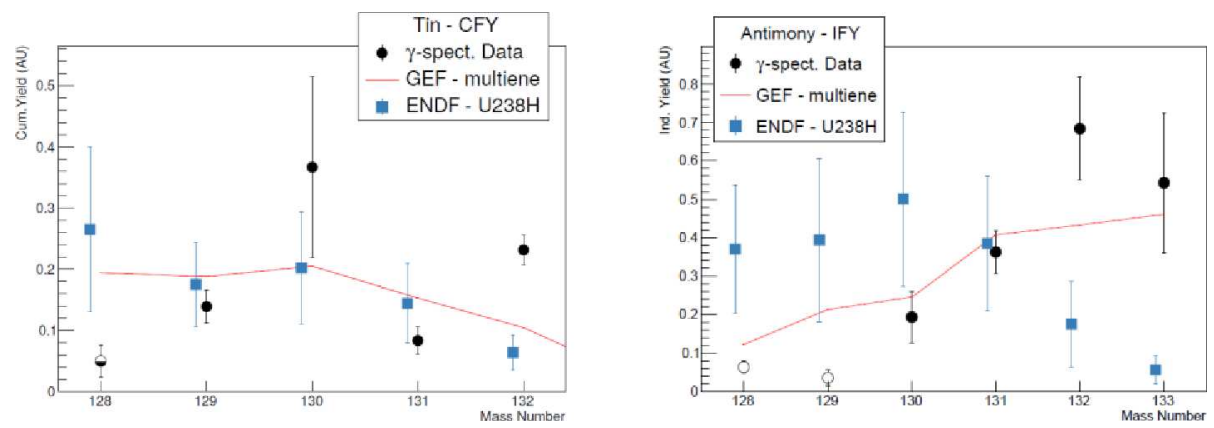

Figure 2. Preliminary results for relative cumulative FY for Sn (left) and independent FY for Sb (right) in the ${ }^{n a t} \mathrm{U}(\mathrm{n}, \mathrm{f})$ reaction. The semi-open circle indicates that only the ground state is included in the data point, and the open circles indicate that only the isomer could be measured. The solid red line represents calculations using the GEF code weighted with the neutron energy spectrum. The blue squares are evaluated data from ENDF/B-VII at $14 \mathrm{MeV}$.

flux of more than $2 \cdot 10^{12} \mathrm{n} / \mathrm{sr} / \mathrm{s}$ can be achieved with a $100 \mu \mathrm{A}$ proton current. The neutron flux with energies above $1 \mathrm{MeV}$ would actually be $2-3 \cdot 10^{12} \mathrm{n} / \mathrm{sr} / \mathrm{s}$ [11].

The data presented here were, however, obtained with the K-130 cyclotron and a proton current of only $1 \mu \mathrm{A}$ since the MCC30/15 was not available during the measurement campaign in December 2016. This meant that we could not achieve sufficient intensities to get good measurement statistics using JYFLTRAP. Instead the fission products were directed to a $\gamma$-spectrometry station where the presented results were obtained. Nevertheless, the measured data here are the first $(\mathrm{n}, \mathrm{f})$ data measured at IGISOL.

According to the characterization data the effective neutron energy spectrum is rather wide. The effective neutron energy, determined by weighted averaging of the neutron energy spectrum with the fission cross section for the ${ }^{n a t} \mathrm{U}$ target, is $12.4 \pm 8.8 \mathrm{MeV}$. About $(97 \pm 1) \%$ of all fission events are from ${ }^{238} \mathrm{U}(\mathrm{n}, \mathrm{f})$. According to calculations with the GEF code [20], first chance fission contributes with 55\%, second chance with $31 \%$, third and fourth chance fission with 8 and $6 \%$, respectively. The pre-fission emission of other particles is negligible. We could also measure the ratio of the $1+/ 2+$ charge states. For Sn we obtain $1.19 \pm 0.17$ and for $S b 2.78 \pm 0.42$. It should be noted, however, that the different probabilities to reach the $1+$ state do not affect the results presented below.

Here we summarize the preliminary results from the December 2016 campaign. A more detailed publication has been submitted [21]. Figure 2 shows the measured relative fission yields for Sn and $\mathrm{Sb}$ in the mass range between 128 and 133.

The measured FY for Sn are cumulative. The measured FY for Sn data can be subtracted from the $\mathrm{Sb}$ data and, therefore, the $\mathrm{Sb}$ yields are independent FY. The figure shows a comparison of the measurement results to evaluated data and calculations using the GEF code. Note that we normalize to the area under the respective distributions since our measured data are not on an absolute scale. The discussion is, therefore, based on the observed shapes only. In the Sn case, we observe a structure with a relatively low yield for ${ }^{131} \mathrm{Sn}$ and a high yield in ${ }^{132} \mathrm{Sn}$. This is not predicted by GEF or present in the evaluated data. The yield for the Sb isotopes is peaked at mass 132. The trend in the yields is roughly reproduced by GEF but is in sharp contrast to the data in the ENDF evaluation which shows a peak in the yield at mass 130 . 
Table 2. Isomeric yield ratios in the ${ }^{n a t} \mathrm{U}(\mathrm{n} . \mathrm{f})$ reaction as obtained in the present measurement compared to calculations with the GEF code and the ENDF/B-VII and JENDL-4.0 evaluations.

\begin{tabular}{|l|l|l|l|l|c|c|}
\hline & $\begin{array}{l}\text { Spin } \\
\text { g.s. }\end{array}$ & $\begin{array}{l}\text { Spin } \\
\text { isom. }\end{array}$ & This work & GEF & ENDF/B-VII.1 & JENDL 4.0 \\
\hline${ }^{129} \mathrm{Sn}$ & $3 / 2^{+}$ & $11 / 2^{-}$ & $\mathbf{0 . 3 7} \pm \mathbf{0 . 1 3}$ & 0.63 & $0.15 \pm 0.12$ & $0.68 \pm 0.43$ \\
\hline${ }^{130} \mathrm{Sn}$ & $0^{+}$ & $\left(7^{-}\right)$ & $\mathbf{0 . 6 4} \pm \mathbf{0 . 4 8}$ & 0.49 & $0.48 \pm 0.49$ & $0.70 \pm 0.46$ \\
\hline${ }^{131} \mathrm{Sn}$ & $\left(3 / 2^{+}\right)$ & $\left(11 / 2^{-}\right)$ & $\mathbf{0 . 4 3} \pm \mathbf{0 . 1 9}$ & 0.69 & $0.48 \pm 0.45$ & $0.81 \pm 0.51$ \\
\hline${ }^{130} \mathrm{Sb}$ & $\left(8^{-}\right)$ & $(4,5)^{+}$ & $\mathbf{0 . 8 1} \pm \mathbf{0 . 4 3}$ & 0.68 & $0.43 \pm 0.41$ & $0.43 \pm 0.28$ \\
\hline${ }^{132} \mathrm{Sb}$ & $\left(4^{+}\right)$ & $\left(8^{-}\right)$ & $\mathbf{0 . 2 5} \pm \mathbf{0 . 1 0}$ & 0.49 & $0.61 \pm 0.41$ & $0.61 \pm 0.20$ \\
\hline
\end{tabular}

Table 2 shows the derived IYR ratio for five cases compared to GEF calculations and evaluated data. Compared to the IYR data in the (p,f) case, the statistical uncertainties are rather large. We expect this to improve considerably when using JYFLTRAP in forthcoming campaigns.

We note that our data are consistent when comparing, e.g., ${ }^{129} \mathrm{Sn}$ and ${ }^{131} \mathrm{Sn}$ where the ground state and the isomer carry the same spin assignments. We also note that the IYR is inversed when comparing the two Sb isotopes. This is consistent with the spin assignments in these two cases which are also inversed between their respective ground state and isomer. Since the evaluations also give large uncertainties it is not possible to observe any clear discrepancy. Also the results from the GEF code are in rough agreement with the measured data. The only exceptions might be ${ }^{129} \mathrm{Sn}$ and ${ }^{132} \mathrm{Sb}$ where the measured IYR seem to be significantly lower than the GEF predictions.

\section{Outlook}

Future work will focus on continued studies on the stopping efficiencies in the fission ion guide and measurement of the transport efficiency of the system using, e.g., ${ }^{252} \mathrm{Cf}(\mathrm{SF})$. This will allow the FY data to be discussed on an absolute scale. We also plan to implement the Phase-Imaging Ion-Cyclotron-Resonance technique to achieve $50 \mathrm{keV}$ mass resolution. This will open up for measurement of a wide range of IYR for many different fissioning systems and a detailed study of the origin angular momentum generation in nuclear fission.

This work has been supported by the European Commission within the CHANDA project (contract 605203), the Swedish Radiation Safety Authority (SSM), and by the Swedish Nuclear Fuel and Waste Management Co. (SKB).

\section{References}

[1] A. Al-Adili et al., these proceedings

[2] S. Julien-Laferrière et al., these proceedings

[3] A. Bail, et al., Phys. Rev. C 84, 034605 (2011)

[4] H. Naik, et al., Nucl. Phys. A 941, 16 (2015)

[5] S. Eliseev, et al., Phys. Rev. Lett. 110, 082501 (2013)

[6] I. Stetcu, et al., Phys. Rev. C 88, 044603 (2013)

[7] A.A. Sonzogni, et al., Phys. Rev. Lett. 116, 132502 (2016)

[8] F.P. An, et al., Phys. Rev. Lett. 118, 251801 (2017)

[9] H. Penttilä, et al., Eur. Phys. J. A 44, 147 (2010)

[10] D. Gorelov, et al., Nucl. Inst. Meth. Phys. Res. B 376, 46 (2016) 
[11] A. Mattera, et al., Eur. Phys. J. A 53, 173 (2017)

[12] A. Al-Adili, et al., Eur. Phys. J. A 51, 59 (2015)

[13] P. Helgesson and H. Sjöstrand, in manuscript

[14] H. Penttilä, et al., Eur. Phys. J. A 52, 104 (2016)

[15] V. Rakopoulos, et al., to appear in Eur. Phys. J. (Proceedings of ND2016)

[16] V. Rakopoulos, et al., in manuscript

[17] M. Tanikawa, et al., Z. Phys. A 347, 53 (1993)

[18] I.V. Ryzhov, et al., Phys. Rev. C 83, 054603 (2011)

[19] A. Solders, et al., Nucl. Data Sheets 119, 338 (2014)

[20] K.-H. Schmidt, et al., Nucl. Data Sheets 131, 107 (2016)

[21] A. Mattera, et al., submitted to Eur. Phys. J. 
